\title{
Research on Frequency Adaptability of Photovoltaic Power Generation
}

\author{
He Tingyi $^{1}{ }^{*}$, Li Shengnan ${ }^{1}$, Wu Shuijun ${ }^{2}$, Meng xian ${ }^{1}$, Mu runzhi ${ }^{2}$ \\ ${ }^{1}$ The Electric Power Research Institute in Yunnan Electric Power Grid, Kunming, Yunan, 650217, China \\ ${ }^{2}$ Electric Power test and Research Institute in Yunnan, Kunming, Yunan, 650217, China
}

\begin{abstract}
In this paper, photovoltaic power generation(PV) and the asynchronously grid-connected power grid are taken as the research objects, and the frequency adaptability of PV to power grid is studied. The influence mechanism of grid frequency variation on PV is revealed, and it is proposed that the frequency tolerance range of PV is mainly determined by the setting value of inverter protection and PLL parameters. The whole process simulation of wind turbine adaptability under frequency change is realized on Matlab/Simulink, and the simulation results verify the correctness of the conclusion.
\end{abstract}

\section{Introduction}

After asynchronous networkin between Yunnan power grid and China Southern Power Grid, the system capacity becomes smaller and the anti-disturbance ability becomes worse[1-2]. The issue of frequency has replaced the transient stability issue as its main problem[3]. By 2019, the installed capacity of renewable energy in Yunnan has reached 11.57 million kilowatts, and in the dry season, the highest proportion of power generation in some regions has reached nearly $50 \%$. With the change of power grid characteristics and high proportion of the renewable energy grid connected [4-6], the adaptability of renewable energy to frequency has become a problem that must be studied.

Based on the MATLAB / Simulink platform, this paper mainly studies frequency adaptability of PV. Through a large number of theoretical research and simulation verification, it is concluded that the response characteristics and performance parameters of PLL will affect the response characteristics of PV when the frequency changes, reasonable setting of inverter protection setting value and PLL parameters can make the grid frequency change has little impact on the photovoltaic generation.

\section{Mathematical model of photovoltaic power generation}

The control strategy of PV is generally vector control based on voltage orientation. Considering the control system design, it is necessary to refer to the mathematical model of inverter based on synchronous coordinate axis shown in formula (1). In voltage oriented control, the daxis of synchronous rotating coordinate axis coincides with the grid voltage vector E, so that the space vector of fundamental wave is a constant component, while other harmonic space vectors have pulsating components. The purpose of grid connected inverter is to output standard sinusoidal current, which requires the reference current component based on synchronous coordinate axis to be direct current. The output current vector diagram of grid connected inverter based on grid voltage orientation is shown in Figure 1.

$$
\left\{\begin{array}{l}
L_{g} \frac{d i_{g d}}{d t}=-R_{g} i_{g d}+m_{d} U_{\mathrm{dc}}-e_{g d}+\omega_{g} L_{g} i_{g q} \\
L_{g} \frac{d i_{g q}}{d t}=-R_{g} i_{g q}+m_{q} U_{\mathrm{dc}}-e_{g q}-\omega_{g} L_{g} i_{g d} \\
C \frac{d U_{\mathrm{dc}}}{d t}=\frac{3}{2}\left(-m_{d} i_{g d}-m_{q} i_{g q}\right)+i_{p v}
\end{array}\right.
$$

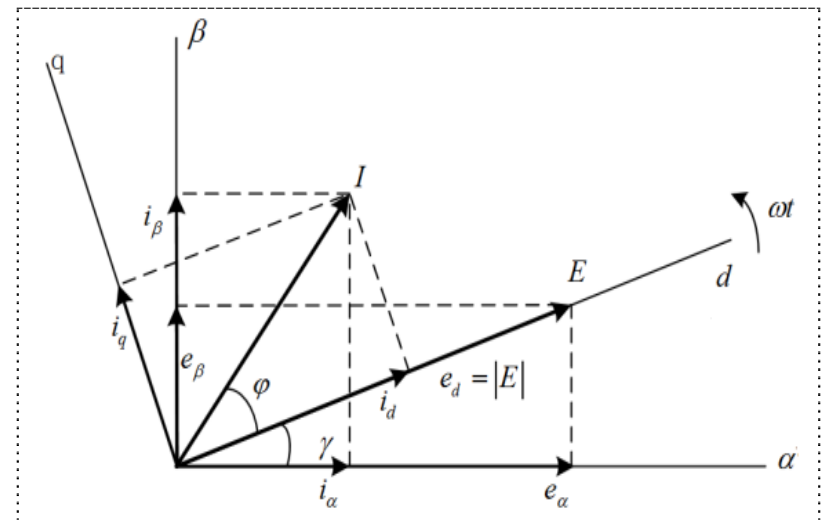

Figure 1. Vector control based on grid voltage orientation

The active power and DC bus voltage can be adjusted by controlling the $\mathrm{d}$-axis component of reference current. The reactive power can be controlled by controlling the qaxis component of reference current. Generally, in order 
to ensure the operation of unit power factor, the current vector and grid voltage vector should be in the same phase. According to the instantaneous power theory, the active and reactive power generated by grid connected inverter is shown in formula(2).

$$
\left\{\begin{array}{l}
p=\frac{3}{2}\left(e_{d} i_{d}+e_{q} i_{q}\right) \\
q=\frac{3}{2}\left(e_{d} i_{q}-e_{q} i_{d}\right)
\end{array}\right.
$$

Due to the vector orientation according to the grid voltage, the above formula can be simplified to formula(3).

$$
\left\{\begin{array}{l}
p=\frac{3}{2} e_{d} i_{d} \\
q=\frac{3}{2} e_{d} i_{q}
\end{array}\right.
$$

According to formula(3), the active power and reactive power are proportional to the dp axis component $i_{d}$ and $i_{q}$ of the inverter reference current. When the grid voltage remains unchanged, The output power of grid-connected inverter can be adjusted by adjusting the voltage of the grid.

In the PV shown in Figure 1, the instantaneous value of active power is $P=i_{p v} u_{d c}$. Without considering the time delay effect of the DC capacitor element and the loss of the inverter, the power on both sides of the DC bus is $P=i_{p v} u_{d c}=3 / 2 e_{d} i_{d}$. Energy loss in power grid voltage is constant and ignore the inverter, the output current of PV maintain constant value, $u_{d c}$ is proportional to the inverter output power, so the DC bus voltage can be controlled through the control of $i_{d}$. The control block diagram of vector control system based on grid voltage orientation is shown in Figure 2

\section{The response characteristics of PV under grid frequency changes}

In actual operation, the grid frequency always gradually decreases or increases at different rates. In general, the grid frequency change rate is much lower than the maximum tracking rate of the phase-locked loop. The PLL can quickly enter the lock after a short capture process, and then it has been tracking during the grid frequency change process. Estimate the frequency and The changing grid frequency is consistent. Therefore, the PV can work normally.

The following figure shows the simulation of the positive and negative sequence fundamental components, phase angles and fundamental frequency components of the grid voltage under the condition of the grid frequency gradually changing; the frequency gradually rises at 1 . $5 \mathrm{~Hz} / \mathrm{s}$ at $100 \mathrm{~ms}$ and continues to 2 . $1 \mathrm{~s}$. If the phase-locked loop can track the grid frequency changes in time and accurately, the PV should work normally. At this time, the frequency and phase lock effect of SSRF-SPLL is shown in Figure 3.
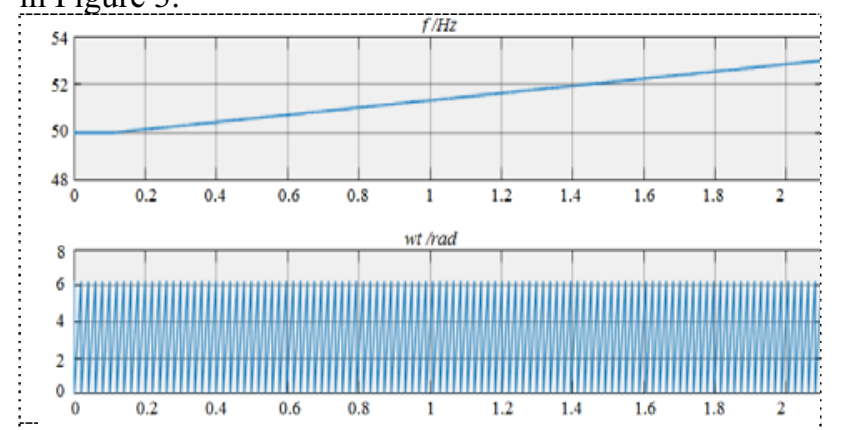

Figure 3. Comparison of frequency and phase when frequency changes 
It can be seen from Figure 3 that when the grid frequency gradually rises at $1.5 \mathrm{~Hz} / \mathrm{s}$, the phase-locked loop can still track the grid frequency in real time without error. The gradual change of grid frequency has little effect on PV. PV can withstand a large range of slow changes in frequency, and its limit is affected by the combined effect of under-over-frequency protection and PLL performance.

\section{Simulation analysis}

Using MATLAB/Simulink, this paper combines the power grid frequency variation range of Yunnan power grid after the occurrence of ac/dc locking fault, and dynamically simulates and validates the response characteristics of PV.

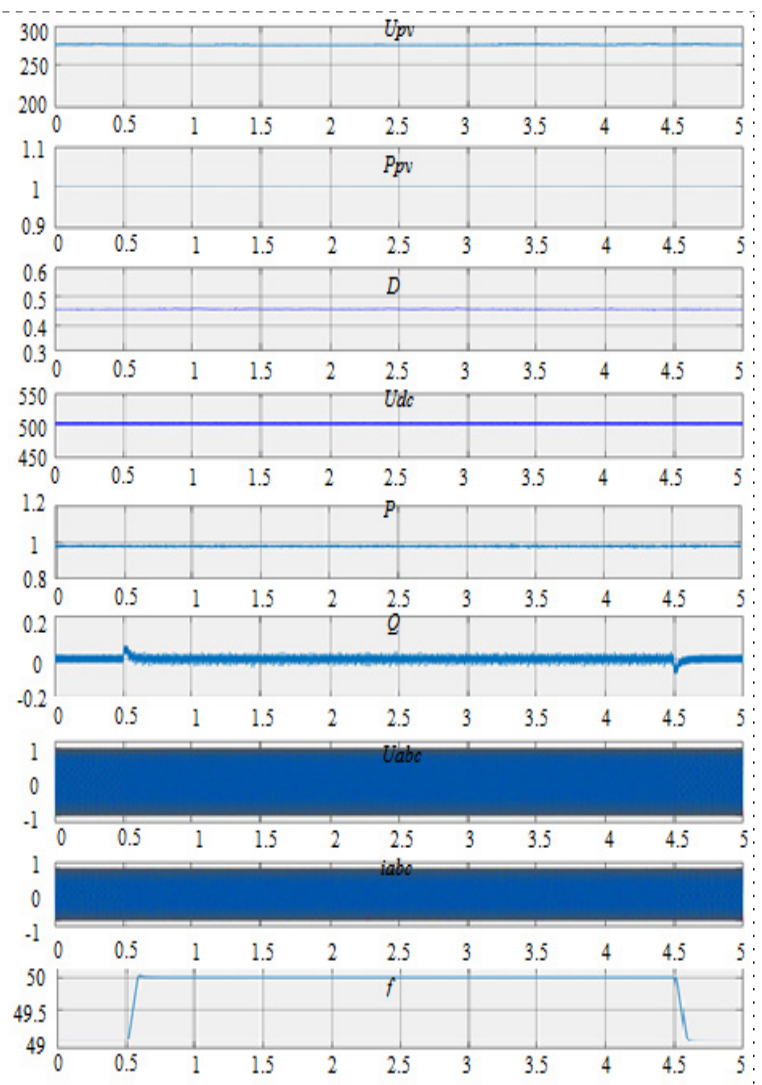

Figure 5. Simulation of frequency down step 


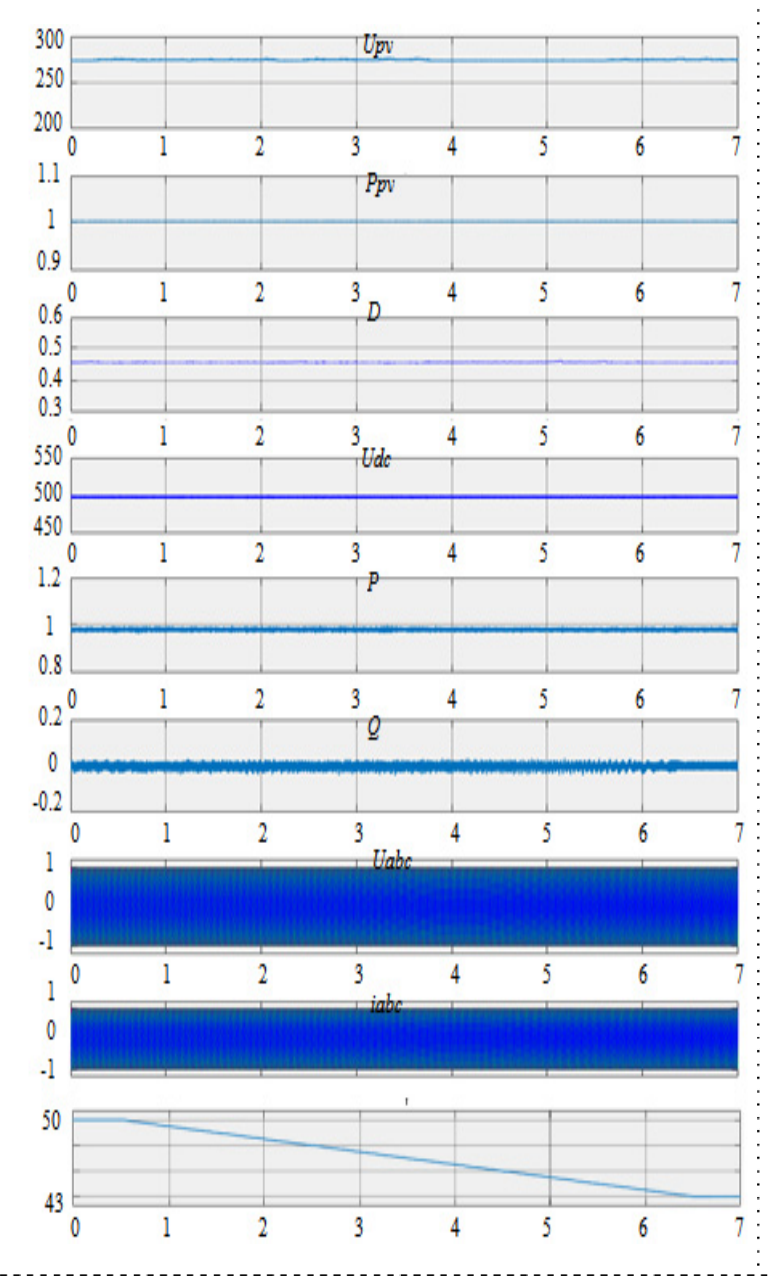

Figure 6. Decrease $7 \mathrm{~Hz}$ at $1 \mathrm{~Hz} / \mathrm{s}$

It can be seen from Figure 4 to Figure 7 that PV can track frequency changes quickly and accurately. After frequency changes, the grid-connected current can reach a steady state relatively quickly, and the DC voltage is stable with almost no change. It shows that the electrical response characteristics of $\mathrm{PV}$ are different from traditional generation and other renewable energy. It is not restricted by rotational inertia, and AC and DC electrical quantities can quickly respond to the target value. When the grid frequency changes suddenly, the active power output of PV remains unchanged. Compared with the
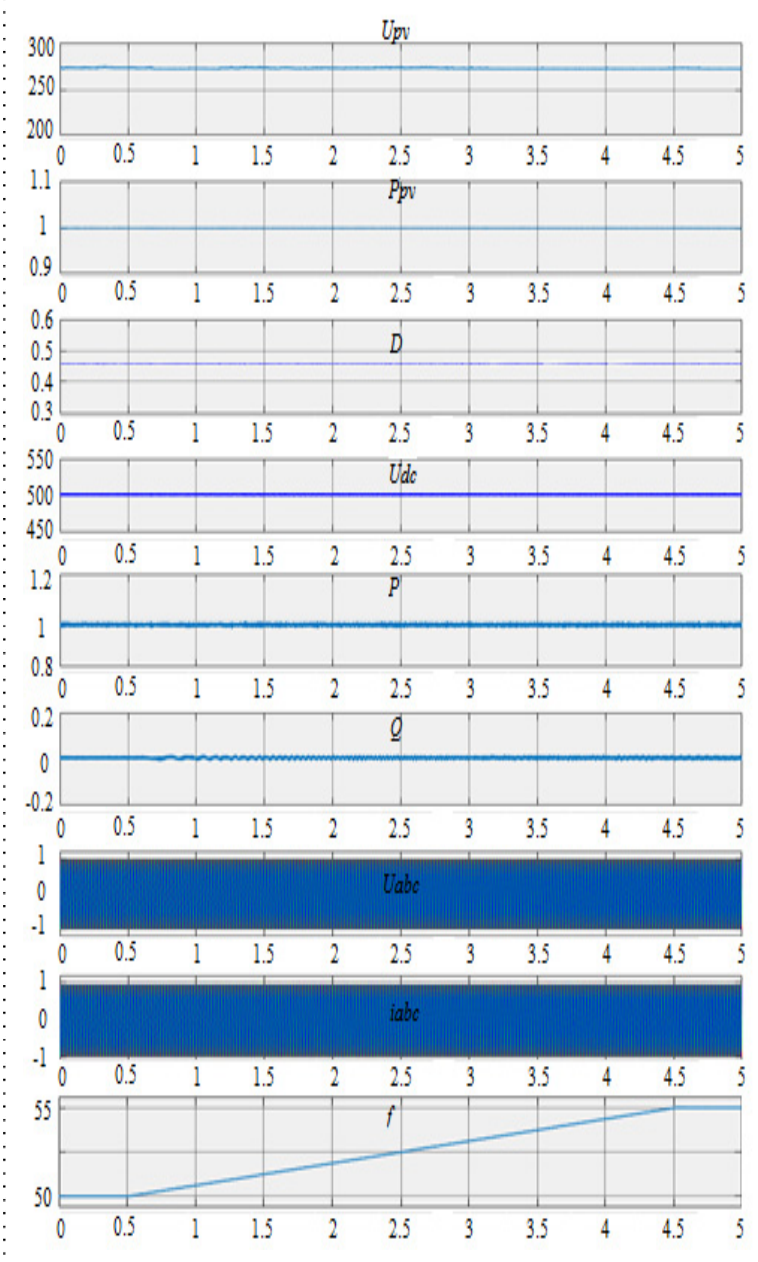

Figure 7. Increase $5 \mathrm{~Hz}$ at $1 \mathrm{~Hz} / \mathrm{s}$

gradual change in frequency, the reactive power fluctuation of the PV is smaller and can be restored in a short time. In summary, the grid frequency changes, PV can operate normally.

When frequency suddenly changes, there is a small reactive power fluctuation. In order to study the impact of frequency changes on PVs under different conditions, considering the extreme conditions of frequency changes, a simulation analysis of the frequency adaptability of PVs under different light intensity and temperature conditions was carried out. 


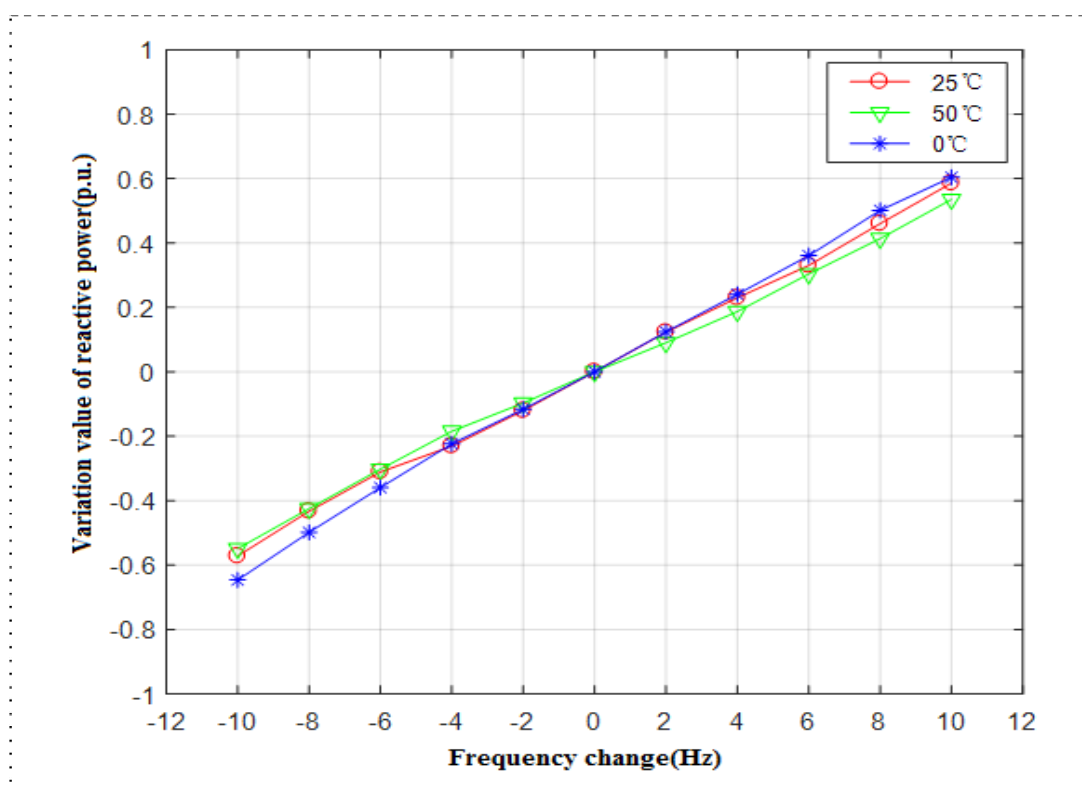

Figure 8. Simulation of frequency changes at $1000 \mathrm{~W} / \mathrm{m} 2$ at different temperatures

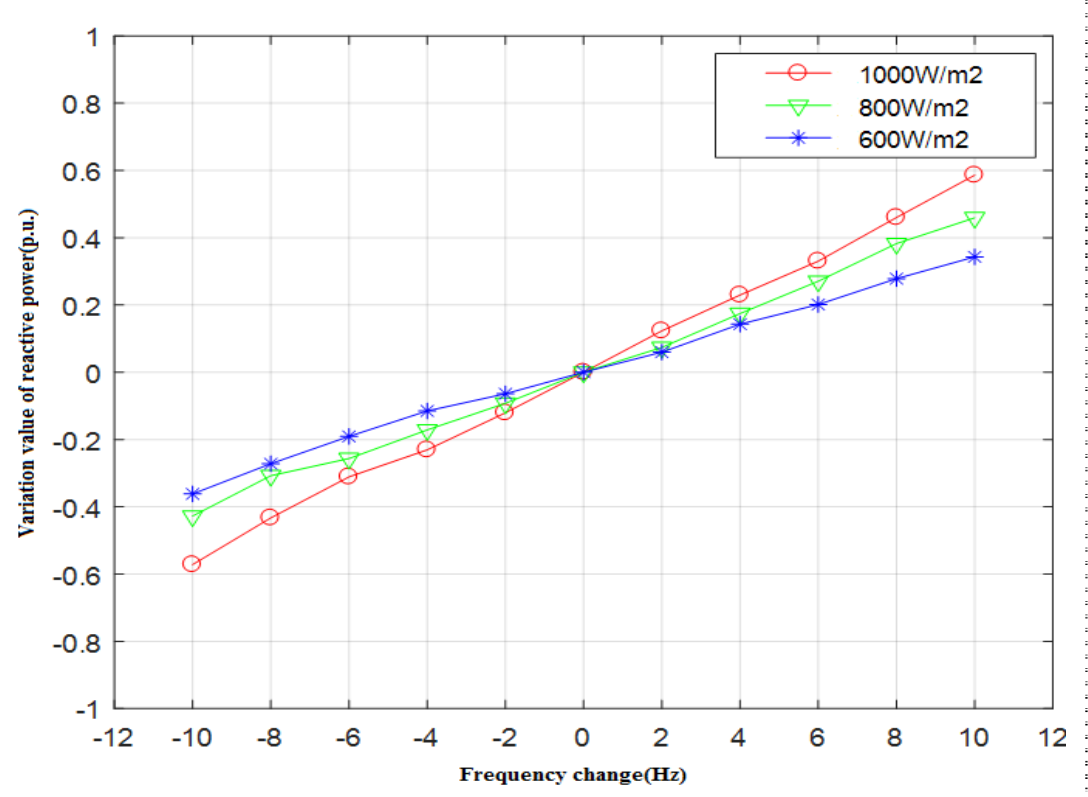

Figure 9. Simulation of frequency change under different light intensity at $25^{\circ} \mathrm{C}$

According to the characteristics of photovoltaic cells, when the temperature decreases, the output power will increase. Under the same light intensity, the disturbance amplitude of reactive power fluctuation is the largest at $0^{\circ} \mathrm{C}$. At $50^{\circ} \mathrm{C}$, the reactive power fluctuation decreases, but the amplitude is basically the same, as shown in Figure 9. The greater the grid frequency changes, the greater the reactive power fluctuation. However, the power change caused by the frequency change will disappear in a short time, as shown in Figure 10. Therefore, the sudden change in frequency has less impact on the photovoltaic system.

\section{Conclusion}

Frequency changes have little impact on PV, and the frequency limit is determined by the inverter protection settings and PLL parameters. And because the inverter over/under frequency protection range is much smaller than the PLL operating limit, the inverter frequency protection limit determines the frequency variation range within which PV can operate normally. Based on this, different power grids can ensure the frequency adaptability of photovoltaic inverters by setting corresponding inverter protection limits and PLL parameters according to the characteristics of their respective power grids.

\section{Acknowledgments}

This article is one of the phased achievements of China Southern Power Grid's scientific and technological project 
"Research on Renewable Energy Participation in Grid Frequency Regulation of Regional Power Grid" (YNKJXM20191240).

\section{References}

1. Du Bin, Zhang dan, Zhang Junmin, Xu Min, Liu Yongjun, TU Liang, FU Chao, WU Xiaoshan, (2016)Stability Characteristics and Control Measures of Yunnan Power Grid After Asynchronous Interconnection, Southern Power System Technology, 7:13-16, 23

2. FU Chao, Liu Yongjun, TU Liang, Li peng, Hong chao, Wu chen, Xu Min, Zhao rui, (2016)Experiment and Analysis on Asynchronous Interconnection System of Yunnan Power Grid and Main Grid of China Southern Power Grid, Southern Power System Technology, 7:2428

3. Cai Baorui, Wang Xingang, Si Dajun, (2015)Impact of Asynchronous Networking for Yunnan Power Grid Security and Stability, Yunnan Electric Power, 1:83-86

4. CHEN Yiping, LI Chongtao, YANG Ruopu, ZHANG Yong, TANG Zhuoyao, (2020)Analysis Method of Piecewise Linear Model for Frequency Oscillation in Asynchronous Power Grid of Yunnan in China, Automation of Electric Power Systems, 44(6), 186-193

5. CHEN Yiping, LI Chongtao, YANGRuopu, ZHANG YongTANG, Zhuoyao, (2020)Analysis Method of Piecewise Linear Model for Frequency Oscillation in Asynchronous Power Grid of Yunnan in China, Automation of Electric Power Systems, 44(6)

6. XIAO Youqiang, YU Qiuyang, CHEN Yixuan(2018)Research on Oscillation Characteristics and Out-of-step Separation System Scheme for Yunnan Power Grid After Asynchronous Interconnection, Jiangsu Electrical Engineering, 37(1), 97-102, 108 